\title{
Modeling socially anhedonic syndromes: genetic and pharmacological manipulation of opioid neurotransmission in mice
}

\author{
C Cinque ${ }^{1,2}$, S Pondiki ${ }^{1,2}$, D Oddi ${ }^{1,2}$, MG Di Certo ${ }^{1,2}$, S Marinelli ${ }^{1,2}$, A Troisi $^{3}$, A Moles $^{1,2,4}$ and FR D'Amato ${ }^{1,2}$
}

Social anhedonia, or the diminished capacity to experience pleasure and reward from social affiliation, is a major symptom of different psychiatric disorders, including some forms of infantile autism and schizophrenia spectrum disorders. The brain opioid hypothesis of social attachment is a promising model for achieving insights into how neurobiological and developmental factors contribute to the regulation of social reward. In this study, genetic knocking-out and naltrexone (NTRX) treatment during the first 4 days of life were used to disrupt opioid neurotransmission in mouse pups and their attachment relationships with the mother. Both permanent (genetic) and transient (pharmacological) manipulations of opioid neurotransmission exerted long-term effects on social affiliation. When juveniles, both $\mu$-opioid receptor knockout mice and NTRX-treated pups showed reduced interest in peers and no preference for socially rewarding environment. These results demonstrate that sociability in juvenile mice is highly dependent on the establishment during infancy of a positive affective relationship with their mothers and that opioid neurotransmission has a major role in the regulation of social hedonic capacity. If the validity of this animal model will be confirmed by future research, translational studies focusing on the interaction between early experience and opioid neurotransmission could provide useful insights for identifying endophenotypes of human psychiatric disorders associated with social anhedonia.

Translational Psychiatry (2012) 2, e155; doi:10.1038/tp.2012.83; published online 28 August 2012

\section{Introduction}

In species with a complex social life, affiliative interactions represent an important component of the behavioral repertoire. Affiliative patterns vary across species but some behaviors are common to most mammals, including mother-infant contact, grooming, social play, and matingrelated affiliative patterns. Presumably, the adaptive value of affiliative relationships has evolved into a set of physiological mechanisms that predispose social animals to relate to conspecifics and to experience pleasure and positive affect from social contact and relatedness. In human beings, social hedonic capacity, or the ability to experience pleasure and reward from social affiliation, is considered a personality characteristic that is normally distributed in non-clinical populations. ${ }^{1,2}$ A few individuals (notably those with schizoid personality or autistic spectrum disorders) seem to lie at the lower extreme of this continuum, experiencing little or no positive feelings during affiliative interactions. ${ }^{3,4}$

Over the last decades, animal models have achieved enormous insights into how neurobiological factors contribute to the regulation of social reward. Recent animal studies have shown that the neuropeptides oxytocin and ariginine vasopressin could have a specific impact on social approach behavior, social affiliation and social attachment. ${ }^{5,6}$ There is preliminary evidence that both neuropeptides may exert similar effects in human subjects. ${ }^{7,8}$
Another neurobiological system that is likely to be implicated in the modulation of social reward is opioid activity. Originally formulated by Panksepp, ${ }^{9}$ the brain opioid hypothesis of social attachment posits that reductions in opioid activity should increase desire for social companionship, and increases in this system should reduce the need for affiliation. ${ }^{10}$ Observations consistent with this hypothesis have been collected in a large variety of species using several distinct behavioral measures. ${ }^{11}$ Animals treated with moderate doses of opiates tend to socially isolate themselves; conversely, the opioid antagonists naloxone and naltrexone (NTRX) have opposite effects, increasing affiliation.

In a previous study, we demonstrated that a geneticinduced deficit in the $\mu$-opioid system was associated with a defective infant-mother bond in mouse pups. ${ }^{12}$ Compared with intact controls (wild-type; WT), $\mu$-opioid receptor knockout pups $(\mu-\mathrm{KO})$ emitted fewer ultrasonic vocalizations (USVs) when isolated from dam and littermates. Such a reduction in USVs was specific to social isolation and not seen when pups were exposed to cold temperature or male mice odors. In addition, these KO pups showed decreased preference/ability to discriminate their mother's scent and no potentiation of callings after repeated separation from the mother. Taken together, these data suggest that endogenous opioids binding to $\mu$-opioid receptors are important for the formation of infants' attachment bond in mice. In line with

${ }^{1} \mathrm{CNR}$, Cell Biology and Neurobiology Institute, Roma, Italy; ${ }^{2}$ RCCS Santa Lucia Foundation, Roma, Italy; ${ }^{3}$ Department of Systems Medicine, University of Rome Tor Vergata, Roma, Italy and ${ }^{4}$ Genomnia, Linate, Italy

Correspondence: Dr FR D’Amato, CNR, Cell Biology and Neurobiology Institute, via del Fosso di Fiorano 64/65, Roma 00143, Italy.

E-mail: francesca.damato@cnr.it

Keywords: affiliation; animal model; attachment behavior; naltrexone; ultrasonic vocalizations; $\mu-\mathrm{KO}$ mice

Received 7 March 2012; revised 25 June 2012; accepted 5 July 2012 
these findings, Roth and Sullivan ${ }^{13,14}$ reported similar results in neonatal rats, showing that NTRX, an opioid antagonist, was able to prevent acquisition, consolidation and expression of odor preference during the sensitive period.

The aim of the present study was to confirm and extend our previous findings showing an opioid-related early attachment deficit and ascertain whether it translates into long-lasting alterations of social behavior. In the first part of the study, we compared pups and young $\mu-\mathrm{KO}$ with aged matched WT animals on a battery of behavioral tests designed to evaluate attachment behavior and to assess affiliation and social reward. We decided to focus on juveniles because human clinical syndromes characterized by pervasive social deficits (for example, autism and schizophrenia spectrum disorders) generally manifest during adolescence and respond better to early interventions. ${ }^{15,16}$

In the second part of the study, we aimed to ascertain whether the behavioral alterations observed in young $\mu-\mathrm{KO}$ mice were the consequence of abnormal mother-infant interactions or whether they reflected a permanent malfunctioning of the opioid system. To test these alternative hypotheses, we induced a reversible pharmacological blocking of the opioid system with NTRX, ${ }^{17}$ during the first postnatal days, to prevent the formation of mother-infant bond in outbred mice. The specificity of such a bond is based on mutual recognizing capability. ${ }^{12,18}$ The rationale for using NTRX requires an explanation. In fact, whereas NTRX in adult animals induces a transient increase in social affiliation, including the display of attachment-related behaviors, ${ }^{19}$ administration of NTRX in pups that have not yet formed an attachment bond with their mothers interferes with the neurochemical opioid mechanisms of social reward. ${ }^{20}$ We hypothesized that such experimental manipulation would impede the formation of the infant-mother attachment and cause social behavioral modifications later in life.

\section{Materials and methods}

Animals, housing conditions and breeding procedures. Orpm $+/+(\mathrm{WT})$ and Orpm $-/-(\mu-\mathrm{KO})$ mice were used to confirm and investigate the role of a genetic deletion of $\mu$-opioid receptors on sociability in youth (experiment 1 ); NMRI outbred mice (Harlan, Harlan, Italy) were used to investigate the role of a pharmacological-induced deficit in attachment during early life on subsequent sociability (experiment 2). The generation of mice lacking $\mu$-opioid receptors was previously described. ${ }^{21} \mathrm{WT}$ and $\mu-\mathrm{KO}$ subjects of these experiments derived from homozygous breeding pairs. NMRI mice used in this study arrived in our lab when 5-6 weeks old and were housed in groups of four in transparent high-temperature polysulfone cages $\left(27 \times 21 \times 14 \mathrm{~cm}^{3}\right)$ with water and food available ad libitum. Room temperature $\left(21 \pm 1^{\circ} \mathrm{C}\right)$ and a $12: 12 \mathrm{~h}$ lightdark cycle (lights on at 1900 hours) were kept constant.

In both experiments the mating protocol consisted in housing one male with two females for 15 days. Pregnant females were isolated around delivery and cages were inspected twice a day for pups: the day of birth was considered postnatal day 0 (PND0). Weaning was performed between PND28 and PND30: animals of similar age/sex and genotype were housed in groups of 2-5 per cage.
Another group of NMRI mice born and reared under standard conditions in our animal facility were used as stimulus partners. All animal used procedures were in strict accordance with standard ethical guidelines (European Community Guidelines on the Care and Use of Laboratory Animals $86 / 609 / E E C)$ and the Italian legislation on animal experimentation (Decreto L.vo 116/92).

Experimental design. In the first experiment USVs and preference for mother's cues were measured in WT and $\mu$ KO pups, to replicate previous data. The relevance and the response to social stimuli were evaluated in juvenile mice in the social approach-avoidance test ${ }^{22,23}$ or in a social placeconditioning paradigm. ${ }^{24}$

In the second experiment a total of 22 NMRI litters were used. On PND1 litters were culled to eight pups (four males and four females). From PND1 to PND4 half of each litter (two males and two females) was injected twice a day (0800 and 1800 hours) with NTRX (1 $\left.\mathrm{mg} \mathrm{kg}^{-1}, \mathrm{sc}\right)$, the other half was saline (SAL) injected. After the first injection all pups (NTRX and SAL) were tattooed. USVs, preference for mother's cues, and response to social stimuli were measured in NTRX- and SAL-treated NMR1 mice by the social approach-avoidance and place preference conditioning test.

\section{Behavioral measures}

Pups' attachment behavior. Attachment behavior was measured in pups by USVs during separation from the mother, and by homing behavior, that is, measuring capability/ motivation to orient towards their mother's cues. USVs were measured on PND8. Vocalizations uttered by isolated pups in either (i) a beaker containing home-cage bedding, or (ii) a beaker containing clean bedding, were recorded for $5 \mathrm{~min}$ using Avisoft technology (Supplementary Information). No more than four pups per litter were tested. After the test, pup's body weight and sex (and tattoo for NMR1 mice) were noted. Homing behavior was measured in PND10 pups. The capability of pup to orient towards familiar odorous cues was evaluated in a small apparatus $\left(5 \times 33 \times 10 \mathrm{~cm}^{3}\right)$ with a central Plexiglas part $\left(5 \times 5 \mathrm{~cm}^{2}\right.$, starting point $)$ that separated, with sliding doors, two differently scented chambers, one covered with pups' home-cage bedding, the other covered with bedding from the cage of un unknown mother with its litter (same postnatal day and genetic background). After 1 min of habituation in the central part of the maze, the lateral doors were opened and the pup could move freely in the apparatus for $5 \mathrm{~min}$. The test was video-recorded and the time spent in the compartments was evaluated thereafter. No more than four pups per litter were tested.

Social approach-avoidance test. Animals were tested immediately before weaning in a gray Plexiglas rectangular box $\left(60 \times 40 \times 24 \mathrm{~cm}^{3}\right)$ consisting of three interconnected chambers. $^{22,23}$ Two identical clear Plexiglas cylinders $(8 \mathrm{~cm}$ in diameter) with multiple small holes were placed, one in each end chamber of the apparatus. During the habituation session ( $5 \mathrm{~min}$ ) the mouse was placed in the central chamber and allowed to freely explore the whole apparatus. A stimulus NMRI mouse, age/sex matched, was then introduced into one cylinder (pseudo-randomly chosen) for $5 \mathrm{~min}$, whereas a 
white object was introduced into the other cylinder. Both sessions were recorded and the time the subject mouse spent in each chamber was measured by a video-tracking system (Smart 1.1). After each test, the entire apparatus was carefully cleaned with $10 \%$ ethanol. Habituation preference scores were measured to evaluate a priori discrimination between lateral compartments (Supplementary Information) and animals were scored for sociability in the test session (sociability index: $100 \times$ time in social chamber/(time in social chamber + time in non-social chamber).

Rewarding value of social interactions. Behavioral testing was conducted as previously described ${ }^{24}$ according to a Conditioned Social Place Preference protocol (CSPP). Briefly for the first $24 \mathrm{~h}$ following weaning (PND28-30) two males and two females from different litters, but same genotype, were housed together in a cage that contained a set of novel environmental cues (social housing condition). For the next $24 \mathrm{~h}$ mice were socially isolated within a second, distinct home-cage environment (isolated housing condition). The two conditions differed for the bedding used. Following the completion of 10 conditioning sessions the CSPP response of each mouse was evaluated (Supplementary Information). The preference score was evaluated as follow: CSPP index $=100 \times$ time social-cued chamber/(time socialcued chamber + time-isolated cued chamber).
Statistical analysis. USVs parameters were evaluated by a three-way analysis of variance, the factors being the genotype/treatment, the sex of the pups and the experimental condition (clean or mother/nest bedding). Two-way analysis of variances, factors being genotype/treatment and sex, were used for homing, approach-avoidance and placepreference tests.

\section{Results}

Effects of genetic deletion of the $\mu$-opioid receptor gene on social anhedonia. Confirming the results of our previous study, $\mu-K O$ pups showed a deficit in their attachment behavior $^{12}$ as shown by USVs data indicating that knockout pups did not discriminate between clean and mother/nest bedding during isolation (Figure 1a). By contrast, WT pups strongly reduced callings in the presence of their mother/nest odor, suggesting a calming effect of mother's cues (Tukey post-hoc: $P<0.05)$. This result is based not only on the number and mean duration of calls, but also on frequency modulations (Table 1). As for their approach/preference towards their mother/nest bedding, contrary to WT, $\mu-\mathrm{KO}$ pups showed lower preference, both in terms of time and number of entries into the familiar arm (\%time: $F(1 /$ $64)=4.82, \quad P<0.05 ;$ \%entries: $F(1 / 64)=4.51, \quad P<0.05$, Figure $1 \mathrm{~b})$. Both measures of infant-mother bond were not a

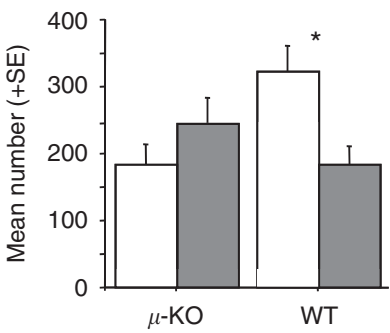

USVs during 5 min of isolation

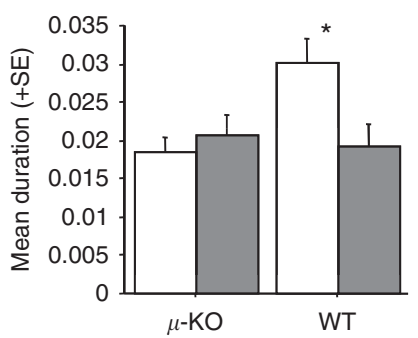

Recognition of own mother/nest cues

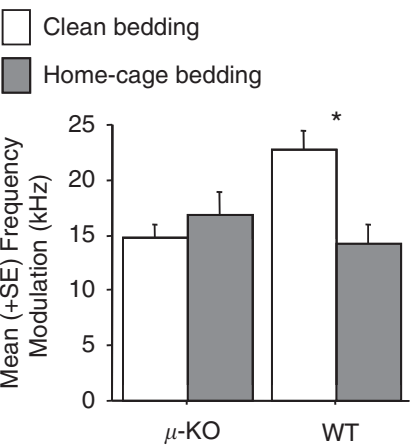

WT
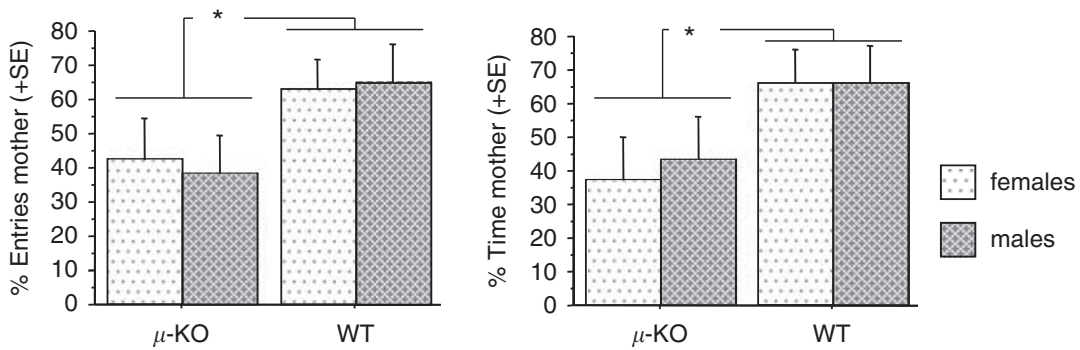

Figure 1 Attachment behavior in knockout pups $(\mu-K O)$ and wild-type (WT) pups. (a) Mean number, duration and frequency modulation (difference between the highest and the lowest peak frequency within each element) of ultrasounds emitted by 8-day old pups during 5 min of isolation in a clean or home-cage bedding beaker. WT pups exposed to clean bedding significantly differed from $\mu-K O$ pups isolated in clean bedding and from WT pups tested in their mother/best bedding. Sample sizes: $\mu$-KO pups in clean bedding: $N=18$, in home-cage bedding: $N=15$; WT pups in clean bedding: $N=18$, in home-cage bedding: $N=15$. (b) Percentage of entries ( $100 \times$ entries mother's compartment/(entries mother's compartment + entries unknown mother-litter compartment) and time (100 $\times$ time mother's compartment/(time mother's compartment + time unknown mother-litter compartment) spent by 10-day old pups in the compartment scented by their mother/nest bedding versus a compartment containing bedding from an unknown mother with its litter. WT pups significantly preferred their mother/nest compartment, whereas $\mu$-KO pups did not. Sample sizes: $\mu-K O$ male pups: $N=15$, female pups: $N=13$; WT male pups: $N=18$, female pups: $N=22 .{ }^{*} P<0.05$. 
affected by sex of the pup and no genotype $\times$ bedding interaction reached significance.

Compared with control animals, adolescent $\mu$-KO mice showed reduced interest in social partners and diminished conditioned social place preference (Figure 2). In the approach-avoidance apparatus, WT mice showed higher sociability in comparisons with $\mu-\mathrm{KO}$ animals, spending more time in the social compartment (Figure 2a: genotype: $F(1 / 41)=9.92, \quad P<0.01)$. No other significant effects emerged. In the social place preference test (Figure 2b), $\mu$ $\mathrm{KO}$ mice avoided the compartment containing cues previously associated with social housing condition whereas the WT mice showed no preference between compartments (genotype: $F(1 / 46)=7.86, P<0.01)$. No other effect reached the significance level.

\section{Effects of postnatal treatment with an opioid antagonist} on social anhedonia in NMR1 mice. The analysis of USVs revealed that overall, NTRX pups did not differ for the number of calls from SAL-treated pups, but only the latter were able to discriminate between clean and mother/nest beddings (treatment: $F(1 / 40)=0.45$, NS; bedding: $F(1 /$ $40)=6.25, P<0.05$; treatment $\times$ bedding: $F(1 / 40)=4.42$, $P<0.05)$ emitting more calls in the absence of familiar cues (Tukey post-hoc: $P<0.05$, Figure 3a). Exposure to mother/

Table 1 ANOVA results for mean number, duration and frequency modulation of ultrasonic vocalizations emitted by $\mu-\mathrm{KO}$ and WT 8-day old pups during $5 \mathrm{~min}$ of isolation in clean or home-cage bedding

\begin{tabular}{lcccc}
\hline & $d f$ & $\begin{array}{c}\text { Mean } \\
\text { number }\end{array}$ & Men duration & $\begin{array}{c}\text { Mean frequency } \\
\text { modulation }^{\mathrm{a}}\end{array}$ \\
\hline Genotype (G) & $1 / 58$ & $\mathrm{~F}=1.19$ & $\mathrm{~F}=2.99$ & $\mathrm{~F}=1.84$ \\
Bedding (B) & $1 / 58$ & $\mathrm{~F}=1.29$ & $\mathrm{~F}=2.43$ & $\mathrm{~F}=3.18$ \\
$\mathrm{G} \times \mathrm{B}$ & $1 / 58$ & $\mathrm{~F}=6.58^{\star}$ & $\mathrm{F}=5.08^{\star}$ & $\mathrm{F}=9.90^{\star \star}$ \\
Sex (S) & $1 / 58$ & $\mathrm{~F}=0.49$ & $\mathrm{~F}=0.11$ & $\mathrm{~F}=2.66$ \\
$\mathrm{G} \times \mathrm{S}$ & $1 / 58$ & $\mathrm{~F}=0.05$ & $\mathrm{~F}=0.02$ & $\mathrm{~F}=0.13$ \\
$\mathrm{~B} \times \mathrm{S}$ & $1 / 58$ & $\mathrm{~F}=0.42$ & $\mathrm{~F}=0.02$ & $\mathrm{~F}=0.32$ \\
$\mathrm{G} \times \mathrm{B} \times \mathrm{S}$ & $1 / 58$ & $\mathrm{~F}=0.56$ & $\mathrm{~F}=0.03$ & $\mathrm{~F}=0.02$ \\
& & & &
\end{tabular}

Abbreviations: ANOVA, analysis of variance; KO, knockout pups; WT, wild-type ${ }^{a} F r e q u e n c y$ Modulation: difference between the highest and the lowest peak frequency within each element. ${ }^{\star} P<0.05,{ }^{\star \star} P<0.01$. nest bedding was responsible for shorter duration of USVs, independently from the pharmacological treatment (treatment: $F(1 / 40)=0.30$, NS; bedding: $F(1 / 40)=6.92$, $P<0.05$; treatment $\times$ bedding: $F(1 / 40)=0.90$, NS). No sex differences emerged for these parameters. The temporary block of opioid receptors also determined reduced preference for mother/nest bedding (Figure 3b: \%time mother: treatment: $F(1 / 34)=6.16, \quad P<0.05 ; \quad \%$ entries mother: treatment: $F(1 / 34)=7.80, P<0.01)$. No Sex and no sex $\times$ treatment effect reached the statistical significance. Overall, these results indicate that NTRX treatment disrupted pup's attachment bond preventing ultrasonic emotional response discrimination between home-cage and familiar bedding during isolation and abolishing selective preference orientation towards mother/nest stimuli.

SAL mice tested for sociability at weaning (Figure 4a) showed higher scores than NTRX mice, suggesting a greater interest towards conspecifics (treatment: $F(1 / 30)=8,81$, $P<0.01$ ). No sex and no sex $\times$ treatment effect reached the significant level. Similar results were obtained in the CSPP test: juvenile NTRX mice showed lower preference for the social-cued chamber in comparison with SAL-treated mice (Figure 4b: Treatment: $F(1 / 20)=23.22, P<0.001$ ). No sex and no sex $\times$ treatment effect reached the significant level.

\section{Discussion}

The attachment behavior system, that is, the behavioral system that promotes proximity to the caretaker maximizing infant survival, influences and organizes motivational, cognitive, emotional and memory processes. ${ }^{25}$ These processes are organized during early infancy with respect to significant caregiving figures and extend into adulthood including attachment to intimate adult partners in humans. Different brain substrates of infant-mother attachment have been identified, the opioid system being one of the possible mediators. ${ }^{11}$ We have already demonstrated ${ }^{12}$ that mice lacking $\mu$-opioid receptors show deficits in two independent measures of attachment behavior: knockout pups were not able to selectively approach their mothers, and did not vocalize during maternal separation. These $\mu-K O$ pups a Interest in social partner

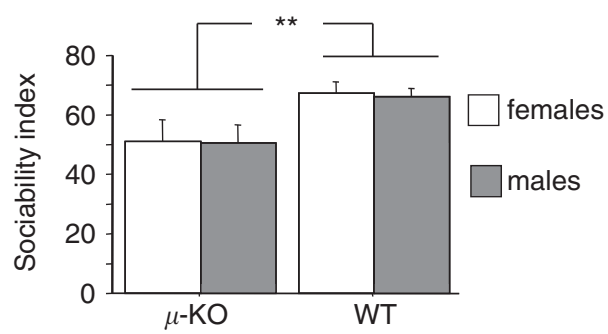

b Conditioned social place preference

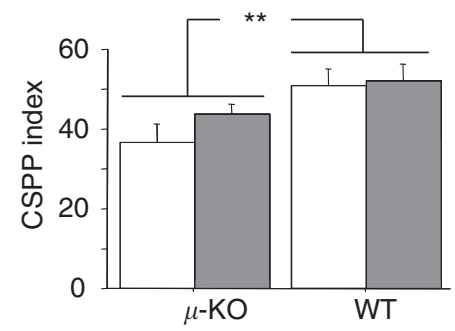

Figure 2 Social behavior characterization of juvenile wild-type (WT) and knockout pups ( $\mu-K O$ ) mice. (a) WT mice significantly prefer the chamber of the apparatus characterized by the presence of a same sex/age partner, contrary to $\mu-\mathrm{KO}$ mice that did not discriminate between the two end chambers. Sociability index: $100 \times$ time in

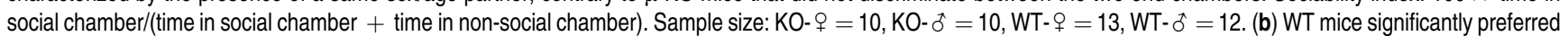
the social-cued chambers of a place preference apparatus, after a 10-day period of conditioning, contrary to KO mice showing no preference between compartments. Conditioned Social Place Preference protocol (CSPP) index $=100 \times$ time social-cued chamber/(time social-cued chamber + time-isolated cued chamber). Sample sizes:

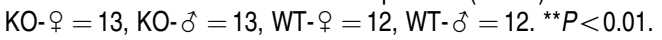


a

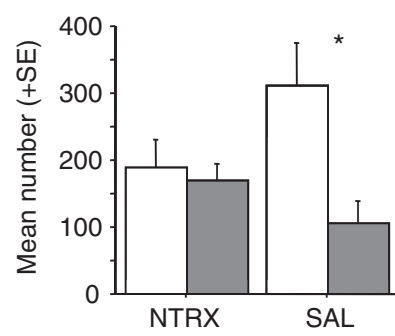

USVs during 5 min of isolation

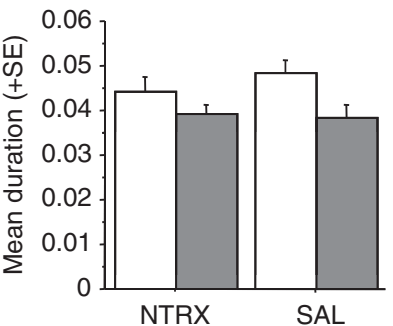

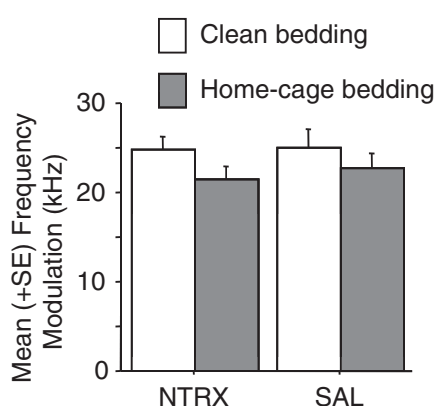

b Recognition of own mother/nest cues
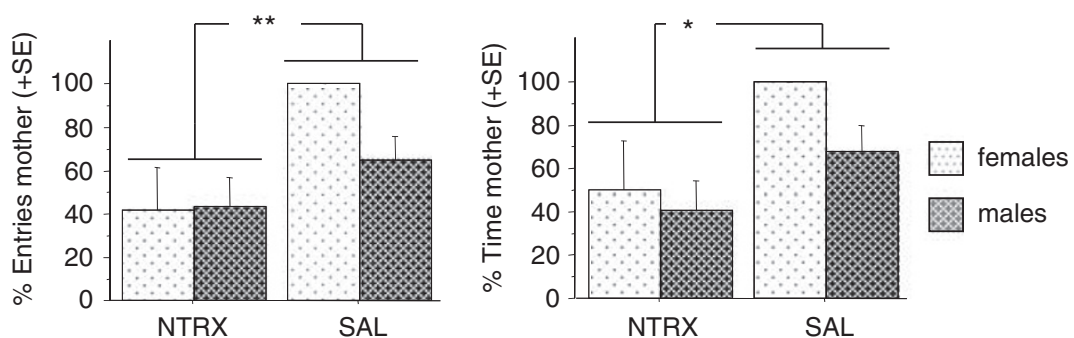

Figure 3 Effects of postnatal naltrexone (NTRX) treatment on NMRI pups' indices of attachment behavior during development. (a) Mean number, duration and frequency modulation (difference between the highest and the lowest peak frequency within each element) of ultrasounds emitted by 8-day old pups during 5 min of isolation in a clean or home-cage bedding beaker. NTRX treatment prevents discrimination of nest versus clean bedding only on the basis of mean number of ultrasonic vocalizations (USVs). Sample sizes: NTRX pups in clean bedding: $N=10$, in home-cage bedding: $N=12$; SAL pups in clean bedding: $N=12$, in home-cage bedding: $N=10$. (b) Percentage of entries (100 $\times$ entries mother's compartment/(entries mother's compartment + entries unknown mother-litter compartment) and time (100 $\times$ time mother's compartment/ (time mother's compartment + time unknown mother-litter compartment) spent by 10-day old pups in the compartment scented by their mother/nest bedding versus a compartment containing bedding from an unknown mother with its litter. SAL pups significantly preferred their mother/nest compartment, whereas NTRX pups did not. Sample sizes: NTRX male pups: $N=12$, female pups: $N=6$; WT male pups: $N=14$, female pups: $N=6$. ${ }^{*} P<0.05 ;{ }^{*} P<0.01$.

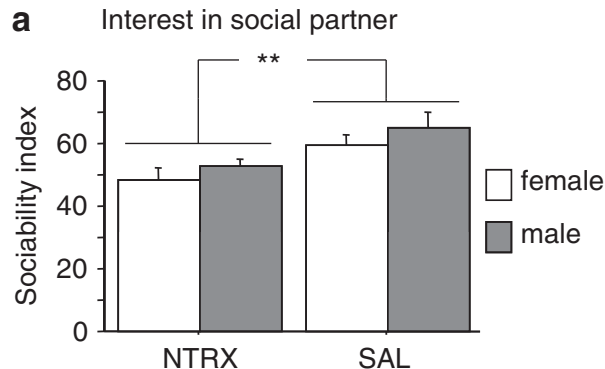

b Conditioned social place preference

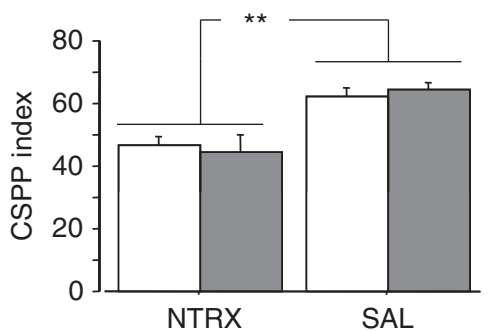

Figure 4 Social behavior characterization of juvenile naltrexone (NTRX) postnatally treated mice. (a) SAL mice significantly prefer the chamber of the apparatus characterized by the presence of a same sex/age partner, contrary to NTRX mice that did not discriminate between the two end chambers. Sociability index: $100 \times$ time in social chamber/(time in social chamber + time in non-social chamber). Sample size: NTRX- $+=9, \mathrm{NTRX}-\hat{\sigma}=9, \mathrm{SAL}-\rho=7, \mathrm{SAL}-\hat{\sigma}=9$. (b) SAL mice significantly preferred the social-cued chambers of a place preference apparatus, after a 10-day period of conditioning, contrary to NTRX mice showing no preference between compartments. Conditioned Social Place Preference protocol (CSPP) index $=100 \times$ time social-cued chamber/(time social-cued chamber + time-isolated cued chamber). Sample size: $\mathrm{SAL}-q=7, \mathrm{SAL}-\hat{0}=5$, NTRX- $+=6$, NTRX- $-{ }^{\star}=6 .{ }^{\star \star} P<0.01$.

seemed to be specifically less sensitive to the absence of maternal cues. The etiology of this insensitivity is likely due to a diminished capacity to experience pleasure and reward related to maternal stimuli. The mesocorticolimbic reward system has been implicated in social attachment and Roth and Sullivan's experimental studies ${ }^{13,14,20}$ support the hypothesis that the $\mu$-opioid system is a crucial component of the social reward processes modulating the positive affective states associated with maternal stimuli. ${ }^{26,27}$

The aim of this study was to investigate whether, according to Panksepp's suggestion, ${ }^{28}$ deficits in infant-mother attachment could affect the development of later social interactions. In line with our hypothesis, we found that juveniles' social motivation and emotional profiles are altered in mice showing dysfunctional attachment in infancy. This finding was confirmed by manipulating the $\mu$-opioid system functioning in two different ways: permanent genetic knocking-out or temporary pharmacological blocking during the first postnatal days.

Compared with our previous experiment, ${ }^{12}$ in the present study we used more sophisticated equipment for the analysis of ultrasounds. $\mu-\mathrm{KO}$ mice showed higher frequency 
modulation than WT pups and this could explain the greater number of USVs detected in this study. The mean number, duration and frequency modulation of USVs were strongly affected by experimental condition in WT pups, whereas $\mu$-KO infants did not modify their calls according to environmental cues. In addition, no differences between male and female pups in both measures of attachment behavior were detected.

To ensure comparability with our previous experiment, ${ }^{12}$ we used knockout and WT mice derived from homozygous lines. Such a strategy of subject selection implied that not only pups but also their mothers had different genetic profiles. Thus, to exclude maternal effects on pups' attachment behavior, we carried out a detailed analysis of mothers' behavior during the first week of life (Supplementary Figure 1) indicating that $\mu-\mathrm{KO}$ and WT dams did not differ as for maternal behaviors directed towards their pups.

The results of the first experiment support Panksepp's hypothesis that both motivation for, and reward from, social stimuli are reduced in $\mu-\mathrm{KO}$ animals. Juvenile $\mu-\mathrm{KO}$ mice showed not only no preference for the social chamber and reduced sociability in comparison with WT peers, but also social place-conditioning avoidance. Importantly, based on data collected during the habituation session, mice did not differ for their general exploratory performance, but they reacted differently to social stimuli.

The second experiment was conducted to ascertain if altered social motivation and reward in juveniles depended on dysfunctional infant-mother attachment, or alternatively on abnormal opioid neurotransmission affecting knockout animals during their entire life. Blocking the opioid receptors, NTRX treatment prevents the formation of infant-mother bond. NTRX-treated pups did not increase vocalization rate in the absence of their mothers' cues and did not discriminate between their mothers' and an unknown dam's bedding. These effects are unlikely to depend on the acute effects of NTRX. NTRX is a short half-life drug $^{29}$ and there was a 4-6day interval between the last injection (PND4) and behavioral tests (PND8-10). Thus, these effects are likely to result from a deficit in early bond formation due to the NTRX block of the $\mu$ opioid component of the reward system.

As for the long-term effects on social behavior of this early sub-chronic treatment, we did find permanent deficits in juveniles. In particular, we found a reduced interest in social partners by NTRX animals. Confirming this, NTRX mice also showed no social conditioned place preference, contrary to SAL mice that spent more than $60 \%$ of their time in the chamber containing cues previously associated to social housing.

Taken together, the results of these experiments suggest that sociability in juvenile mice is highly dependent on the establishment of a positive affective relationship with their mothers during infancy. Both permanent and temporary disruption of the opioid reward circuit during infancy reduced affiliative behaviors during adolescence. Our findings suggest the existence of a very narrow time window, from PND1 to PND4 (NTRX treatment) that has a crucial role in terms of setting the neurobiological basis for social affiliation. It is worth noting that the sub-chronic NTRX treatment did not disrupt social behavior in term of competence or skills, but mainly affected the affiliative motivation and the affective component of social interactions. These alterations are more evident during adolescence and tend to disappear during adulthood. Adult reproductive behaviors as maternal behavior in $\mu-\mathrm{KO}$ mice and exploratory courtship behavior (both in $\mu-\mathrm{KO} / \mathrm{WT}$ and NTRX/SAL mice, (Supplementary Table 2) were unaffected by the genetic and pharmacological treatments. Similarly, reproductive behavior is maintained in BTBR $\mathrm{T} 1 \mathrm{tf} / \mathrm{J}$, a genetic mouse model of autism recently developed, even if these mice show altered social behaviors during dyadic interactions. ${ }^{30}$

Conflicting results concerning the effects of age on sociability are reported in the literature..$^{23,31}$ These contrasting findings might depend on the difficulty to ascertain if social approach reflects affiliative or aggressive motivation. In fact, behavioral tests for assessing sociability are based on spatial proximity, but generally prevent physical contact that could reveal the real nature of the social interaction. A few studies have allowed a free interaction session after the approachavoidance test, and sometimes aggression occurred within 35 min. ${ }^{31,32}$ To minimize the likelihood that social approach was motivated by aggression, in this study we used young/notalready-weaned animals of the same sex/line/treatment of the experimental subject. The evaluation of sociability and affiliation in adult mice, a species characterized by male territoriality and aggression, is somewhat difficult. ${ }^{33}$ In this study, adult animals were observed in a reproductive context (that is, mating and caregiving), and no abnormal behaviors were observed.

The present study has some limitations. First, human variability in opioid functioning does not reach the extreme dysfunctionality induced by genetic knocking-out. In this regard, animal models using subjects with genetic polymorphisms are closer to human variability. ${ }^{34-36}$ Second, s.c. injections represent stressful events per se for mouse pups and, even if we used SAL-injected controls to minimize this problem, changes in physiological and behavioral parameters could have occurred in our subjects.

The results of the present study, together with those of previous reports, ${ }^{9,11,12,19}$ may be useful to build an animal model of human psychiatric disorders implying defective social motivation and reduced sensitivity to social reward. Psychiatric conditions with these clinical features include some forms of infantile autism and schizophrenia spectrum disorders. ${ }^{4,37}$ There are several characteristics of the animal model that support its validity as a useful tool to investigate the pathogenesis and treatment of defective social motivation and reduced sensitivity to social reward. First, the model involves dysfunctional attachment processes, and there is evidence that early experience and relationships with caregivers have a role in the vulnerability to social anhedonia and dismissing attachment in human subjects. ${ }^{38}$ Second, a role for altered opioid neurotransmission has been demonstrated in some patients suffering from psychiatric disorders associated with social anhedonia. ${ }^{39}$ Third, natural genetic variation in human subjects (resembling that experimentally-induced in animal models ${ }^{12,34-36}$ ) has been shown to influence social motivation and the capacity to experience pleasure from affiliative interactions. ${ }^{40,41}$ Finally, the emergence of social deficits during childhood (and years before the onset of the clinical symptoms) is a peculiar feature of psychiatric conditions with 
high levels of social disability. ${ }^{42}$ If the validity of the animal model will be confirmed by future research, translational studies focusing on the interaction between early experience and opioid neurotransmission could provide useful insights for identifying endophenotypes of human psychiatric disorders associated with social anhedonia and for developing effective intervention strategies to be implemented early in life.

\section{Conflict of Interest}

The authors declare no conflict of interest.

Acknowledgements. Thanks to Brigitte $L$ Kieffer for comments on the manuscript, scientific support and providing animals. This study has been granted by Telethon, Italy (Grant no. GGP05220) and also partially supported by funds from Regione Lazio for 'Sviluppo della Ricerca sul Cervello'.

1. Brown LH, Silvia PJ, Myin-Germeys I, Kwapil TR. When the need to belong goes wrong: the expression of social anhedonia and social anxiety in daily life. Psychol Sci 2007; 18 : 778-782.

2. Harvey PO, Pruessner J, Czechowska Y, Lepage M. Individual differences in trait anhedonia: a structural and functional magnetic resonance imaging study in non-clinical subjects. Mol Psychiatry 2007; 703: 767-775.

3. Kwapil TR. Social anhedonia as a predictor of the development of schizophrenia-spectrum disorders. J Abnorm Psychol 1988; 107: 558-565.

4. Troisi A, D'Amato FR. Deficit in affiliative reward: an endophenotype for psychiatric disorders? Behav Brain Sci 2005; 28: 365-366.

5. Insel TR, Young LJ. The neurobiology of attachment. Nat Rev Neurosci 2001; 2: 129-136.

6. Skuse DH, Gallagher L. Dopaminergic-neuropeptide interactions in the social brain. Trends Cogn Sci 2009; 13: 27-35.

7. Heinrichs M, Domes G. Neuropeptides and social behaviour: effects of oxytocin and vasopressin in humans. Prog Brain Res 2008; 170: 337-350.

8. Ebstein RP, Israel S, Lerer E, Uzefovsky F, Shalev I, Gritsenko I et al. Arginine vasopressin and oxytocin modulate human social behavior. Ann NY Acad Sci 2009; 1167: 87-102.

9. Panksepp J, Herman B, Conner R, Bishop P, Scott JP. The biology of social attachments: opiates alleviate separation distress. Biol Psychiatry 1978; 13: 607-618.

10. Stein DJ, van Honk J, Ipser J, Solms M, Panksepp J. Opioids: from physical pain to the pain of social isolation. CNS Spectr 2007; 12: 669-674.

11. Nelson EE, Panksepp J. Brain substrates of infant-mother attachment: contributions of opioids, oxytocin, and norepinephrine. Neurosci Biobehav Rev 1988; 22: 437-452.

12. Moles A, Kieffer BL, D'Amato FR. Deficit in attachment behavior in mice lacking the mu-opioid receptor gene. Science 2004; 304: 1983-1986.

13. Roth TL, Sullivan RM. Endogenous opioids and their role in odor preference acquisition and consolidation following odor-shock conditioning in infant rats. Dev Psychobiol 2001; 39: $188-198$

14. Roth TL, Sullivan RM. Consolidation and expression of a shock-induced odor preference in rat pups is facilitated by opioids. Physiol Behav 2003; 78: 135-142.

15. Harrop C, Trower P. Why does schizophrenia develop at late adolescence? Clin Psychol Rev 2001; 21: 241-265.

16. Seltzer MM, Krauss MW, Shattuck PT, Orsmond G, Swe A, Lord C. The symptoms of autism spectrum disorders in adolescence and adulthood. J Autism Dev Disord 2003; 33 565-581.

17. Vukojević V, Ming Y, D’Addario C, Hansen M, Langel U, Schulz R et al. Mu-opioid receptor activation in live cells. FASEB J 2008; 22: 3537-3548.

18. Ostermeyer MC, Elwood RW. Pup recognition in Mus musculus: parental discrimination between their own and alien young. Dev Psychobiol 1983; 16: 75-82.

19. Schino G, Troisi A. Opiate receptor blockade in juvenile macaques: effect on affiliative interactions with their mothers and group companions. Brain Res 1992; 576: $125-130$.
20. Roth TL, Sullivan RM. Examining the role of endogenous opioids in learned odor-stroke associations in infant rats. Dev Psychobiol 2006; 48: 71-78.

21. Matthes HW, Maldonado R, Simonin F, Valverde O, Slowe S, Kitchen I et al. Loss of morphine-induced analgesia, reward effect and withdrawal symptoms in mice lacking the mu-opioid-receptor gene. Nature 1996; 383: 819-823.

22. Brodkin ES, Hagemann A, Nemetski SM, Silver LM. Social approach-avoidance behavior of inbred mouse strains towards DBA/2 mice. Brain Res 2004; 1002: 151-157.

23. Moy SS, Nadler JJ, Perez A, Barbaro RP, Johns JM, Magnuson TR et al. Sociability and preference for social novelty in five inbred strains: an approach to assess autistic-like behavior in mice. Genes Brain Behav 2004; 3: 287-302.

24. Panksepp JB, Lahvis GP. Social reward among juvenile mice. Genes Brain Behav 2007; 6: 661-671.

25. Bowlby J. The nature of a child's tie to his mother. Int J Psychoanal 1958; 39: 350-373.

26. Herman BH, Panksepp J. Effects of morphine and naloxone on separation distress and approach attachment: evidence for opiate mediation of social affect. Pharmacol Biochem Behav 1978; 9: 213-220.

27. Panksepp J, Siviy SM, Normansell LA. Brain opioids and social emotions. In: Reite M, Field T (eds) The Psychobiology of Attachment and Separation. Academic Press: New York, 1985. pp 3-49.

28. Panksepp J, Nelson E, Siviy S. Brain opioids and mother-infant social motivation. Acta Paediatr Suppl 1994; 397: 40-46.

29. Alvarez-Fuentes J, Rojas-Corrales MO, Holgado MA, Caraballo I, Micó JA, FernándezArévalo M. Preclinical study of an oral controlled release naltrexone complex in mice. J Pharm Pharmacol 2000; 52: 659-663.

30. Scattoni ML, Ricceri L, Crawley JN. Unusual repertoire of vocalizations in adult BTBR $T+t f / J$ mice during three types of social encounters. Genes Brain Behav 2011; 10: 44-56.

31. Sankoorikal GM, Kaercher KA, Boon CJ, Lee JK, Brodkin ES. A mouse model system for genetic analysis of sociability: $\mathrm{C} 57 \mathrm{BL} / 6 \mathrm{~J}$ versus BALB/cJ inbred mouse strains. Biol Psychiatry 2006; 59: 415-423.

32. Pietropaolo S, Guilleminot A, Martin B, D'Amato FR, Crusio WE. Genetic-background modulation of core and variable autistic-like symptoms in Fmr1 knock-out mice. PLOS ONE 2011; 6: e17073.

33. Bronson FH. The reproductive ecology of the house mouse. Q Rev Biol 1979; 54: 265-299.

34. Barr CS, Schwandt ML, Lindell SG, Higley JD, Maestripieri D, Goldman D et al. Variation at the mu-opioid receptor gene (OPRM1) influences attachment behavior in infant primates. Proc Natl Acad Sci USA 2008; 105: 5277-5281.

35. Higham JP, Barr CS, Hoffman CL, Mandalaywala TM, Parker KJ, Maestripieri D. Mu-opioid receptor (OPRM1) variation, oxytocin levels and maternal attachment in free-ranging rhesus macaques Macaca mulatta. Behav Neurosci 2011; 125: 131-136.

36. Mague SD, Blendy JA. OPRM1 SNP (A118G). involvement in disease development, treatment response, and animal models. Drug Alcohol Depend 2010; 108: 172-182.

37. Kwapil TR. Social anhedonia as a predictor of the development of schizophrenia-spectrum disorders. J Abnorm Psychol 1998; 107: 558-565.

38. Troisi A, Alcini S, Coviello M, Croce Nanni R, Siracusano A. Adult attachment style and social anhedonia in healthy volunteers. Pers Individ Dif 2010; 48: 640-643.

39. Nandhu MS, Naijil G, Smijin S, Jayanarayanan S, Paulose CS. Opioid system functional regulation in neurological disease management. J Neurosci Res 2010; 88: 3215-3221.

40. Troisi A, Frazzetto G, Carola V, Di Lorenzo G, Coviello M, D'Amato FR et al. 2011Social hedonic capacity is associated with the A118G polymorphism of the mu-opioid receptor gene (OPRM1) in adult healthy volunteers and psychiatric patients. Soc Neurosci $2011 ; 6$ : 88-97.

41. Troisi A, Frazzetto G, Carola V, Di Lorenzo G, Coviello M, Siracusano A et al. Variation in the \{micro\}-opioid receptor gene (OPRM1) moderates the influence of early maternal care on fearful attachment. Soc Cogn Affect Neurosci 2012; 7: 542-547.

42. Schiffman J, Walker E, Ekstrom M, Schulsinger F, Sorensen H, Mednick S. Childhood videotaped social and neuromotor precursors of schizophrenia: a prospective investigation. Am J Psychiatry 2004; 161: 2021-2027.

Translational Psychiatry is an open-access journal published by Nature Publishing Group. This work is licensed under the Creative Commons Attribution-NonCommercial-No Derivative Works 3.0 Unported License. To view a copy of this license, visit http://creativecommons.org/licenses/by-nc-nd/3.0/ 